\title{
Úber Kampfgasvergiftungen.
}

\author{
II. Uber Zersetzung der Kampistoffe durch Wasser. \\ Von \\ P. Rona, Berlin.
}

Mit 4 Textabbildungen.

(Eingegangen am 28. Juni 1920.)

Bei der Einatmung von Reizgasen treffen diese auf der feuchten Oberfläche der Luftwege mit Wasser zusammen und treten aus dem gasoder dampfförmigen Zustande in wässerige Lösung über. Es ist daher eine Grundfrage für die Beurteilung ihrer Wirkung, wie weit sie bei der Auflösung in Wasser unverändert bleiben oder zersetzt werden.

Für die Prüfung der Zersetzung der Kampfstoffe kamen zwei Methoden zur Anwendung. Erstens die colorimetrische Bestimmung der Wasserstoffionenkonzentration der Lösung der betreffenden Kampfstoffe in Wasser bzw. der Änderung der H-Konzentration der Lösung oder des Gemisches Kampfstoff-Wasser. Zweitens die Prüfung der Leitfähigkeit während der Einwirkung des Wassers auf den Kampfstoff.

Die erste Methode beruht auf der UUberlegung, daß bei der Zersetzung der meisten Kampfstoffe Säure entsteht, was in einer Erhöhung der Wasserstoffionenkonzentration der Lösung seinen Ausdruck findet. Bei Anwendung geeigneter Indicatoren wird die Farbe der Lösung und namentlich jede Änderung dieser Farbe in dem Umschlagsgebiet des Indicators durch Farbenvergleich mit Lösungen von bekannter $\mathrm{H}$-Konzentration die $\mathrm{H}$-Ionenkonzentration unmittelbar angeben.

Die in der vorliegenden Untersuchung benutzten Indicatoren waren die folgenden: 1 . Neutralrot $(0,1 \mathrm{~g}$ in $500 \mathrm{ccm} 93 \mathrm{proz}$. Alkohol $+500 \mathrm{ccm}$ Wasşer gelöst), 2. Methylrot $(0,1 \mathrm{~g}$ in $300 \mathrm{ccm} 93$ proz. Álkohol + $200 \mathrm{ccm}$ Wasser gelöst), 3. Methylorange $(0,1 \mathrm{~g}$ in $1000 \mathrm{ccm}$ Wasser gelöst), Tropaeolin 00 (0,1 g in $1000 \mathrm{ccm}$ Wasser gelöst). Die Umschlagsgebiete dieser Indicatoren liegen bei folgenden $p_{\mathrm{H}}$ : für 1 . 6,5-8,0, 2. $4,2-6,3,3$. 3,7-4,4, 4. $\left.1,4-2,6^{1}\right)$.

Die zweite Methode fußt darauf, daß die Kampfstoffe im allgemeinen keine Elektrolyte sind; erst bei ihrer Zersetzung entstehen Produkte, die die Leitfähigkeit der (wässerigen) Lösung erhöhen. So kann eine

1) Vgl. L. Mi c h a elis, Die Wasserstoffionenkonzentration. 1914. J. Springer. 
beobachtete Steigerung der Leitfähigkeit als Zeichen und Maß der Zersetzung benutzt werden.

Im einzelnen ergab die Untersuchung folgendes.

\section{Phosgen.}

Von den in Betracht kommenden Methoden ist hier die Indicatorenmethode die geeignetste. Es wurde (nach Sörensen) durch Mischung von Natriumcitrat (0,1 molare Lösung sekundären Natriumcitrats) und Salzsäure $(0,1 \mathrm{n})$ eine abgestufte Wasserstoffionenreihe von 4 Gliedern dargestellt. Die Zusammensetzung der Mischung und die zugehörigen Wasserstoffionenkonzentrationen (ausgedrückt in $p_{\mathbf{H}}$ ) waren die folgenden ${ }^{1}$ ):

\begin{tabular}{|c|c|c|c|c|c|c|}
\hline I. 4,5 & $\mathrm{ccm}$ & Citrat & $+\mathbf{5 , 5}$ & $\mathrm{cem}$ & $\mathrm{HCl}$ & $p_{\mathrm{H}}=3,364$ \\
\hline II. 4,0 & ," & " & $+6,0$ & " & ", & $p_{\mathrm{H}}=2,972$ \\
\hline III. 3,33 & ", & & $+6,67$ & , : & , & $p_{\mathrm{H}}=2,274$ \\
\hline IV. $\mathbf{3 , 0}$ & , & , & $+7,0$ & ,: & ," & $p_{\text {H }}=1,925$ \\
\hline
\end{tabular}

Je $10 \mathrm{ccm}$ dieser Lösungen wurden mit je einem Tropfen Tropaeolin 00 versetzt, das in dem vorliegenden Bereich der Wasserstoffionenkonzentrationen sein Umschlagsgebiet von rot zu gelb hat.

Dann wurden Reagensgläser mit $10 \mathrm{ccm}$ dest. Wasser von $0^{\circ}$ beschickt und mit so viel flüssigem Phosgen (in Aceton gelöst) versetzt, daß die Wasserstoffionenkonzentration der Lösung bei völliger Zersetzung des Phosgens sich in den obigen $p_{\mathrm{H}^{-}}$Grenzen bewegen mußte.

So wurden in einem. Versuch 2 Tropfen flüssiges Phosgen in $10 \mathrm{ccm}$ Aceton getropft (beide Flüssigkeiten standen in Eiswasser), dann wurden vier mit $10 \mathrm{ccm}$ dest. Wasser beschickte, mit je 1 Tropfen Tropaeolin 00 versetzte Reagensgläser (Farbe rein gelb), die im Eiswasser standen, mit bzw. 3, 5, 8, 10 Tropfen der acetonischen Phosgenlösung versetzt.

Es zeigte sich, daß nach dem Zufügen des Phosgens die Wasserstoffionenkonzentration der betreffenden Lösungen sich sofort (der ganze Vorgang des Zufügens und Vermischens des Phosgens dauerte etwa 2-3 Sekunden) bedeutend erhöhte. Der $p_{\mathrm{H}}$-Wert im Reagensglas

Nr. 1 war ca. 3,3
Nr. 2 war zwischen 3,3 und 2,9
Nr. 3 war (fast) 2,9
Nr. 4 war $\quad 2,3$

Ferner ergab sich, daß in der mittels des Indicators gemessenen Wasserstoffionenkonzentration in der Beobachtungszeit von mehreren Stunden (bis zum nächsten Tage) keine Zunahme stattfand. Die gleich am Anfang beobachtete Wasserstoffionenkonzentration war demnach die maximale. Die Zersetzung des Phosgens im Wasser mußte demnach

1) Vgl. S. P. L. Sörense n, Enzymstudien II. Biochem. Zeitschrift \&1, 131 (1909). 
eine äußerst kurz dauernde (momentane) sein, da eine fortschreitende Zunahme der Wasserstoffionenkonzentration auch in den allerersten Beobachtungszeiten nicht nachgewiesen werden konnte.

Orientierende Messungen der Leitfähigkeit des mit Phosgen versetzten Leitfähigkeitswassers sprechen ebenfalls für eine momentane Zersetzung des Phosgens. Wurde von der oben erwähnten acetonischen Phosgenlösung $0,5 \mathrm{ccm}$ zu $20 \mathrm{ccm}$ Leitfähigkeitswasser zugefügt und die Leitfähigkeit gemessen, so sank der Widerstand sofort (d. h. innerhalb der erforderlichen Zeit vom Zufügen der Phosgenlösung bis zur ersten genauen Ablesung des Widerstandes, was $1 / 2-3 / 4$ Minute ausmacht), von ca. $40000 \mathrm{Ohm}$ auf $150 \mathrm{Ohm}$, und blieb dann unverändert auf dieser Höhe.

Verhältnisse im ,künstlichen Blutserum".

Um die Verhältnisse im Blutserum nachzuahmen, wurde ein ,,künstliches" Blutserum durch Mischung von $\mathrm{NaHCO}_{3}$ und $\mathrm{CO}_{2}$ bereitet.

$\mathrm{Zu}$ diesem Zweck wurde eine Lösung von Bicarbonat in der Stärke von 0,13 Mol. im Liter hergestellt und 0,01 Mol. HCl zugefügt. Die zugefügte Säure setzt sich mit dem Bicarbonat um:

$0,13 \mathrm{Mol}$. $\mathrm{NaHCO}_{3}+0,01 \mathrm{Mol}$. $\mathrm{HCl} \rightarrow 0,12 \mathrm{Mol} . \mathrm{NaHCO}_{3}+0,01 \mathrm{Mol} . \mathrm{H}_{2} \mathrm{CO}_{3}$. Die Lösung enthält demnach 0,12 $\mathrm{Mol} . \mathrm{NaHCO}_{3}$ und $0,01 \mathrm{Mol} . \mathrm{H}_{2} \mathrm{CO}_{3}$ pro Liter, ein Verhältnis, das dem im Serum vorhandenen im wesentlichen entspricht. ${ }^{1}$ ) Die Wasserstoffionenkonzentration dieser Lösung ist nahe gleich der des Blutserums, nämlich $0,25 \cdot 10^{-7}$. Das vorliegende Gemisch kann als ein brauchbares vereinfachtes Modell des Blutserums betrachtet werden (Hender son, Mich ael is).

Während im früheren Versuch mit reinem Wasser etwa 1/20 Tropfen Phosgen (auf $10 \mathrm{ccm}$ Wasser) genügte, um die Wasserstoffionenkonzentration auf etwa $p_{\mathrm{H}}=2,9 \mathrm{zu}$ bringen, mußten zu $10 \mathrm{ccm}$ unseres künstlichen Blutserums ca. 5 Tropfen flüssiges Phosgen gefügt werden, um die Wasserstoffionenkonzentration entsprechend zu erhöhen.

Erst nachdem die gesamte Kohlensäure aus dem Bicarbonat durch Phosgen (bzw. durch die daraus entstandene $\mathrm{HCl}$ ) in Freiheit gesetzt worden und gar kein Bicarbonat mehr in Lösung ist, veranlaßt die Salzsäure eine sprunghafte Erhöhung der Wasserstoffionenkonzentration. Bei der Beurteilung der Verhältnisse muß demnach die „Pufferwirkung“ ” des Blutes und der Organsäfte in Betracht gezogen werden.

\section{Uber die Zersetzung des Phosgens im Serum.}

Nach den geschilderten Untersuchungen erfolgt die Zersetzung des Phosgens in Wasser momentan. Es war von Interesse, zu prüfen, ob die Zersetzung des Phosgens im Serum ebenso schnell verläuft. Möglicherweise könnten die Serumkolloide (hauptsächlich die Eiweißkörper des Serums) verlangsamend auf die Zersetzung wirken.

1) Vgl. Henderson, Lawrence, J., Ergebn. d. Physiol. (Asher-Spiro) 8, 254 (1909) und L. Michaelis 1. c. 
Bei der Untersuchung wurde die Leitfähigkeitsmethode angewendet. Als Serum diente ein durch mehrwöchige Dialyse von den Elektrolyten befreites Katzenserum. Alle Versuche wurden bei $0^{\circ} \mathrm{C}$ ausgeführt und während der ganzen Versuchsdauer das Serum im Leitfähigkeitsgefäß energisch gerührt.

1. Ver s uch. Zu $10 \mathrm{ccm}$ Aceton wurden 2 Tropfen Phosgen zugefügt; $20 \mathrm{ccm}$ dialysiertes Serum wurden mit $0,5 \mathrm{ccm}$ dieser Phosgenlösung versetzt.
Widerstand vorher $2100 \Omega$
nach 10 Sekunden 1900 ,"
20 " 1900 ,"
nach 30 Sekunden $1900 \Omega$
$60 \quad 1900$,"
2 Minuten 1900 ,

Der Widerstand bleibt unverändert.

2. Versuch. 10 Tropfen Phosgen in $10 \mathrm{ccm}$ Aceton, davon $1 \mathrm{ccm} \mathrm{zu} 20 \mathrm{ccm}$ dial. Serum.

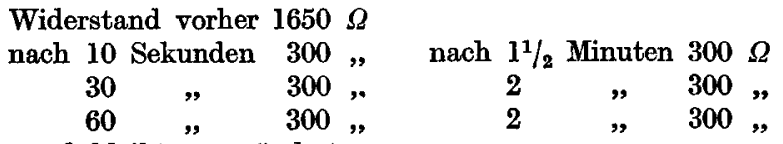

Der Widerstand bleibt unverändert.

3. Versuch. Phosgenlösung wie in Versuch 2; davon $0,25 \mathrm{ccm} \mathrm{zu} 20 \mathrm{ccm}$ dial. Serum.

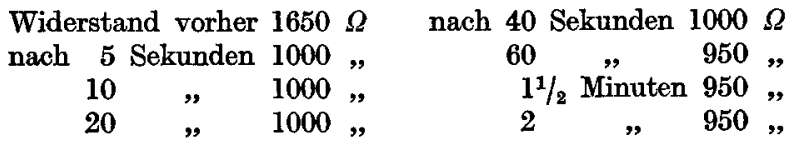

Der Widerstand bleibt unverändert.

Fügt man zum Serum direkt einige Tropfen flüssiges Phosgen zu, so vergehen einige (etwa 25) Sekunden, bis eine völlige Verteilung der spezifisch schwereren Phosgentropfen im Serum erfolgt. Dann bleibt der Widerstand konstant.

4. Vers uch. 2 Tropfen Phosgen werden zu $20 \mathrm{ccm}$ dial. Serum zugefügt.

Widerstand vorher $1600 \Omega$

nach 10 Sekunden 1100 ,"

\begin{tabular}{|c|c|c|c|c|}
\hline 15 & 800, & 60 & ," & 380 \\
\hline 20 & 550, & $1^{1} / 4$ & Minuten & 380 \\
\hline 30 & 400 , & $11 / 2$ & ", & 380 \\
\hline 40 & 380, & 2 & , & 380 \\
\hline
\end{tabular}

Der Widerstand bleibt unverändert.

Aus allen diesen Verșuchen ergibt sich, daß die Zersetzung des Phosgens auch im Serum momentan erfolgt.

\section{Chlorpikrin.}

Chlorpikrin wird durch Wasser nicht zerlegt.

Es wurden folgende Lösungen bzw. Gemische hergestellt:

I. $10 \mathrm{ccm}$ dest. Wasser $+0,1 \mathrm{ccm}$ Chlorpikrin.

II. $10 \mathrm{ccm}$ Aceton $+0,1 \mathrm{ccm}$ Chlorpikrin.

Von dieser Lösung wurden 1. 5 Tropfen

2. 10

3. $15 \quad$ " zu $10 \mathrm{ccm}$ dest. Wasser zugefügt. 
III. $10 \mathrm{ccm}$ Aceton $+0,5 \mathrm{ccm}$ Chlorpikrin.

Von dieser Lösung wurden je 15 Tropfen zu je $10 \mathrm{ccm}$ dest. Wasser zugefügt.

Diese Lösungen gaben mit den angeführten Indikatoren folgende Farben:

$\begin{array}{llcc} & \text { sofort } & \text { nach } 15 \text { Minuten } & \text { nach } 48 \text { Stunden } \\ \text { Lakmus (Papier; blau) } & \text { blau } & \text { blau } & \text { blau } \\ \text { Neutralrot } & \text { gelb } & \text { gelb } & \text { gelb } \\ \text { Methylrot } & \text { gelb } & \text { gelb } & \text { gelb } \\ \text { Methylorange } & \text { gelb } & \text { gelb } & \text { gelb }\end{array}$

Wie man sieht, ist selbst nach 48 Stunden keine Spur von saurer Reaktion nachzuweisen. - Eine Gasbildung unter Einwirkung von Wasser erfolgt nicht.

Löst man $0,5 \mathrm{ccm}$ Chlorpikrin in $10 \mathrm{ccm}$ Aceton und fügt man $1,0 \mathrm{ccm}$ dieser Lösung zu $20 \mathrm{ccm}$ Leitfähigkeitswasser (dessen Widerstand bei ca. $40000 \mathrm{Ohm} \mathrm{lag),} \mathrm{so} \mathrm{beobachtet} \mathrm{man} \mathrm{selbst} \mathrm{nach} \mathrm{mehreren}$ (bis 48) Stunden nur eine äußerst geringe Abnahme des Widerstandes (Zunahme der Leitfähigkeit), die ungezwungen auf zufällige Verunreinigungen des Präparates zurückgeführt werden kann.

\begin{tabular}{|c|c|c|c|c|}
\hline Widerstand & nach & 1 & Minute & $25000 s$ \\
\hline , & , & 5 & Minuten & 25000 \\
\hline , & , & 10 & , & 25000 \\
\hline " & , & 15 & ," & 25000 \\
\hline " & , & 30 & , & 25000 \\
\hline , & , & 60 & , & 25000 \\
\hline , & , & 48 & Stunden & 17000 \\
\hline
\end{tabular}

Die nach Beendigung des Versuchs untersuchte Flüssigkeit gab (mit $\mathrm{AgNO}_{3}$ ) keine Chlorreaktion.

Dichloräthylsulfid (Thiodiglykolchlorid).

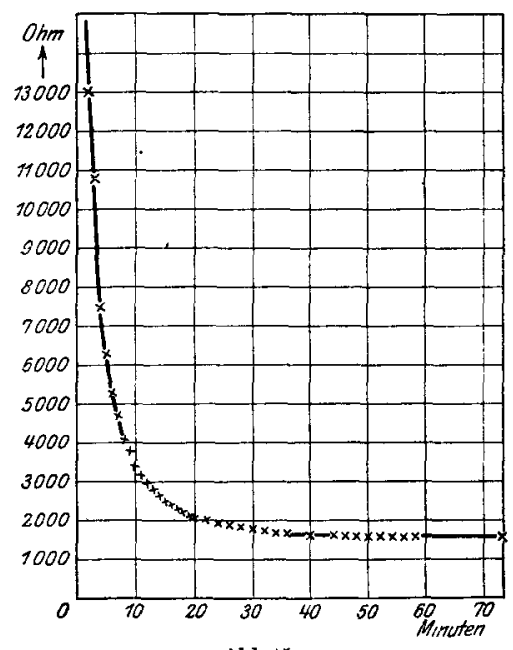

Abb. 1.

Dichloräthylsulfid (Ds) wird von Wasser ganz allmählich zersetzt. Löst man 2 Tropfen Ds in $10 \mathrm{ccm}$ absolutem Alkohol und fügt davon 2-10 Tropfen zu $10 \mathrm{ccm}$ dest. Wasser, so behält die mit Tropäolin gelb gefärbte Lösung zunächst ihre gelbe Farbe und wird erst nach einigen Stunden rot, als Zeichen einer während dieser Zeit zunehmenden Acidität der Lösung.

Genau läßt sich die Zersetzungsgeschwindigkeit von Ds in Wasser mittels Messung der Leitfähigkeit bestimmen. Folgende Versuche in dieser Richtung seien hier angeführt. 
1. Versuch. $0,05 \mathrm{ccm}$ Ds. wurden in $10 \mathrm{ccm}$ absolutem Alkohol gelöst, davon $0,05 \mathrm{ccm} 20 \mathrm{ccm}$ Leitfähigkeitswasser zugefügt und die Widerstände gemessen. Temp. $21^{\circ} \mathrm{C}$.

Widerstand vor dem Zufügen von Ds.: $17000 \Omega$.

\begin{tabular}{|c|c|c|c|c|c|}
\hline nach & 15000 & nach & $22^{\prime}$ & 2050 & $\Omega$ \\
\hline $\mathbf{2}^{\prime}$ & 13000 & ", & $24^{\prime}$ & 1950 & , \\
\hline $3^{\prime}$ & 10000 & , & $26^{\prime}$ & 1870 & , \\
\hline $4^{\prime}$ & 7500 & , & $28^{\prime}$ & 1850 & , \\
\hline $\mathbf{5}^{\prime}$ & 6300 & , & $30^{\prime}$ & 1800 & ", \\
\hline $6^{\prime}$ & 5300 & , & $32^{\prime}$ & 1750 & , \\
\hline $7^{\prime}$ & 4700 & $"$ & $34^{\prime}$ & 1700 & ", \\
\hline $8^{\prime}$ & 4100 & ,, & $36^{\prime}$ & 1700 & , \\
\hline $9^{\prime}$ & 3800 & , & $40^{\prime}$ & 1660 & , \\
\hline $10^{\prime}$ & 3400 & " & $42^{\prime}$ & 1650 & , \\
\hline $11^{\prime}$ & 3200 & , & $44^{\prime}$ & 1650 & , \\
\hline $12^{\prime}$ & 2950 & , & $46^{\prime}$ & 1640 & , \\
\hline $13^{\prime}$ & 2800 & , & $48^{\prime}$ & 1630 & ," \\
\hline $14^{\prime}$ & 2650 & ,, & $50^{\prime}$ & 1625 & , \\
\hline $15^{\prime}$ & 2500 & , & $52^{\prime}$ & 1620 & " \\
\hline $16^{\prime}$ & 2400 & , & $54^{\prime}$ & 1610 & ," \\
\hline $17^{\prime}$ & 2300 & ,, & $56^{\prime}$ & 1600 & , \\
\hline $18^{\prime}$ & 2250 & , & $58^{\prime}$ & 1600 & , \\
\hline $19^{\prime}$ & 2150 & , & $80^{\prime}$ & 1600 & ", \\
\hline $20^{\prime}$ & 2100 & ," & Wide & rstand & nach 4 Tagen $1450 \Omega$. \\
\hline
\end{tabular}

Die vorhandene Ds-Menge wurde demnach in $20 \mathrm{ccm}$ dest. Wasser bei $21^{\circ} \mathrm{C}$ in etwa einer Stunde praktisch vollkommen zerlegt. Graphisch ist der Versuch in der beigefügten Kurve (Abb. 1) wiedergegeben, wo die Abscissen die Zeiten in Minuten, die Ordinaten die gemessenen Widerstände bedeuten. Man sieht, wie die Widerstände mit der Zeit in gesetzmäßiger Weise abnehmen.

Nach Beendigung des Versuches wurden $1 \frac{1}{2}$ Tropfen der Lösung in das Auge einer Katze getropft. Es wurde keine Reizwirkung beobachtet.

2. Ver s u ch. 2 Tropfen Ds. wurden in $2 \mathrm{ccm} 90$ proz. Alkohol ohne Rückstand gelöst. $19 \mathrm{ccm}$ frisch ausgekochtes Leitfähigkeitswasser wurden in ein Elektrodengefä $B$ von der Widerstandskapazität 0,57 gebracht. Der Widerstand das Wassers betrug etwa $50000 \Omega$. $1 \mathrm{ccm}$ der alkoholischen Ds-Lösung wurde zu dem Leitfähigkeitswasser in das Elektrodengefäß gebracht, wobei dieses leicht getrübt wurde. Der Widerstand sank sofort und betrug nach ca. 2 Minuten $1776 \Omega$. Die Temperatur betrug $20^{\circ} \mathrm{C}$. Den weiteren Verlauf des Widerstandes zeigt die Tabelle.

Die Lösung $(20 \mathrm{ccm})$ enthält ca $0,021 \mathrm{~g} \mathrm{Ds}=66,10^{-4} \mathrm{~mol} /$ pro Liter, d. h. $66,10^{-7} \mathrm{~mol} / 1 \mathrm{ccm}$ Gift.

\begin{tabular}{c||c|c|c}
\hline \hline Zeit & Widerstand $\Omega$ & $\begin{array}{c}\text { Leitfahigkeit } \\
10^{4} \varkappa\end{array}$ & $\begin{array}{c}\text { Mol HCl } \mathrm{pro}^{\mathrm{cm}}{ }^{3} \\
10^{6} \eta\end{array}$ \\
\hline $3^{\text {h }} 31^{\prime}$ & 1776,0 & 3,2 & 0,84 \\
$33^{\prime}$ & 832,5 & 6,8 & 1,79 \\
$35^{\prime}$ & 566,1 & 10,0 & 2,90 \\
$37^{\prime}$ & 455,1 & 12,5 & 3,30 \\
$39^{\prime}$ & 385,0 & 14,8 & 3,90 \\
$41^{\prime}$ & 340,0 & 16,8 & 4,42
\end{tabular}


2. Versuch (Fortsetzung).

\begin{tabular}{|c|c|c|c|}
\hline Zeit & Widerstand $\Omega$ & $\begin{array}{c}\text { Leitfähigkeit } \\
104 x\end{array}$ & $\begin{array}{c}\text { Mol pro } \mathrm{cm}^{3} \\
10^{6} \eta\end{array}$ \\
\hline $43^{\prime}$ & 310,0 & 18,4 & 4,89 \\
\hline $45^{\prime}$ & 290,0 & 19,6 & 5,15 \\
\hline $47^{\prime}$ & 273,0 & 20,9 & 5,50 \\
\hline $49^{\prime}$ & 257,0 & 22,2 & 5,89 \\
\hline $51^{\prime}$ & 246,0 & 23,2 & 6,10 \\
\hline $53^{\prime}$ & 236,0 & 24,2 & 6,37 \\
\hline $55^{\prime}$ & 228,0 & 25,0 & 6,58 \\
\hline $57^{\prime}$ & 223,0 & 25,6 & 6,73 \\
\hline $59^{\prime}$ & 219,0 & 26,0 & 6,84 \\
\hline $4^{\mathrm{h}} \mathbf{1}^{\prime}$ & 215,0 & 26,5 & 6,97 \\
\hline $15^{\prime}$ & 200,0 & 28,5 & 7,50 \\
\hline $30^{\prime}$ & 187,0 & 30,5 & 8,00 \\
\hline $45^{\prime}$ & 182,0 & 31,3 & 8,23 \\
\hline $\begin{array}{l}\text { nach wel- } \\
\text { teren } 18 \text { Std. }\end{array}$ & 148,0 & 38,8 & 10,00 \\
\hline
\end{tabular}

3. Versuch. 5 Tropfen Ds $(=0,1068 \mathrm{~g})$ werden in $5 \mathrm{ccm} 90$ proz. Alkohol gelöst, davon $1 \mathrm{ccm}$ zu $19 \mathrm{ccm}$ Leitfähigkeitswasser im Elektrodengefäß (Kapazität 0,57 ) gebracht. Widerstand des Wassers $>50000 \Omega$. Temperatur $19^{\circ} \mathrm{C}$; etwa $2 \mathrm{Mi}$ nuten nach Einbringen des Giftes in das Leitfähigkeitswasser ist der Widerstand auf $3500 \Omega$ gesunken. (Vgl. Abb. 2.)

- Den Verlauf der Zersetzung zeigt folgende Tabelle:

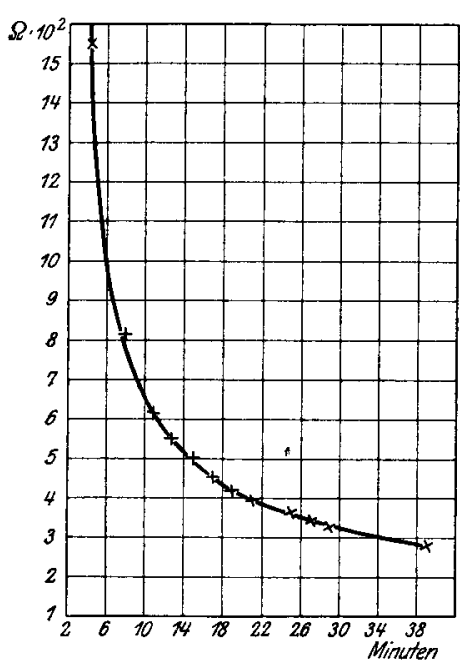

Abb. 2.

\begin{tabular}{|c|c|c|c|}
\hline Zeit & $\begin{array}{c}\text { Widerstand } \\
\Omega\end{array}$ & $\begin{array}{l}\text { Leitfthigkeit } \\
x=10^{4}\end{array}$ & $\begin{array}{c}\text { Mol HCl pro } \\
\text { ccm Lösung } \\
\eta \cdot 10^{\circ}\end{array}$ \\
\hline ln $^{\text {h }} 29^{\prime}$ & 3500 & 1,64 & 0,43 \\
\hline $32^{\prime}$ & 1550 & 3,67 & 0,96 \\
\hline $34^{\prime}$ & 1020 & 5,59 & 1,47 \\
\hline $36^{\prime}$ & 815 & 6,99 & 1,84 \\
\hline $39^{\prime}$ & 615 & 9,27 & 2,44 \\
\hline $41^{\prime}$ & 545 & 10,46 & 2,75 \\
\hline $43^{\prime}$ & 500 & 11,40 & 3,00 \\
\hline $45^{\prime}$ & 455 & 12,53 & 3,30 \\
\hline $47^{\prime}$ & 420 & 13,57 & 3,57 \\
\hline $49^{\prime}$ & 395 & 14,43 & 3,80 \\
\hline $53^{\prime}$ & 367 & 15,53 & 4,09 \\
\hline $55^{\prime}$ & 347 & 16,43 & 4,32 \\
\hline $57^{\prime}$ & 330 & 17,28 & 4,55 \\
\hline $59^{\prime}$ & 320 & 17,93 & 4,72 \\
\hline $2^{\mathrm{h}} \quad 7^{\prime}$ & 282 & 20,21 & 5,32 \\
\hline $13^{\prime}$ & 257 & 22,18 & 5,89 \\
\hline $17^{\prime}$ & 247 & 23,08 & 6,07 \\
\hline $30^{\prime}$ & 225 & 25,35 & 6,68 \\
\hline nach 48 Std. & 204 & 27,94 & 7,35 \\
\hline
\end{tabular}

4. Vers uch. Zur genauen Analyse der Zersetzungskurve wurde der folgende Versuch angestellt (vgl. Abb. 3). 0,1513 g Dichloräthylsulfid wurden. in $7 \mathrm{ccm}$ 90 proz. Alkobol gelöst, $1 \mathrm{ccm}$ davon mit $100 \mathrm{ccm}$ Leitfähigkeitswasser verdünnt. Es entsteht zunächst eine leichte Trübung, die in einigen Sekunden vergeht. 
$100 \mathrm{ccm}$ enthalten demnach $0,0216 \mathrm{~g}$ Ds $=13,6 \cdot 10^{-5}$ Grammoleküle. - Der Widerstand des Leitfähigkeitswassers ist größer als $50000 \Omega$. Gefäßkonstante 0,47. Temperatur $19^{\circ} \mathrm{C}$.

Aus den Widerständen wird nach der Formel $\frac{C}{W}=x$ die Leitfähigkeit berechnet. Unter der bei diesen Konzentrationen gültigen Voraussetzung, daB alles $\mathrm{HCl}$ dissoziiert ist, wird aus $\varkappa$ nach der Formel $\frac{\varkappa}{\eta}=\Lambda_{\infty}(\eta=\mathrm{Mol} \mathrm{HCl}$ pro $1 \mathrm{ccm}$; $\Lambda_{\infty}$ maximale Äquivalentleitfähigkeit der $\left.\mathrm{HCl}=380\right) \eta$ berechnet. Aus $\eta$ unter der Voraussetzung, daß jedes Molekül Ds 2 Atome Cl (gleichzeitig) abgibt, die Menge des zersetzten Giftes pro $1 \mathrm{ccm}$ Lösung $x$ festgestellt. $a$ ist die ursprünglich vorhandene Menge Gift, $a-x$ die jeweils noch vorhandene Menge unzersetzten Giftes.

Es gilt die Formel $\ln \frac{a}{a-x}=k t$

d. h. der Zerfall des Giftes geht nach dem Modus der monomolekularen Reaktion vor sich. $k$ wurde nach der Methode der kleinsten Quadratsummen berechnet zu 0,04298.

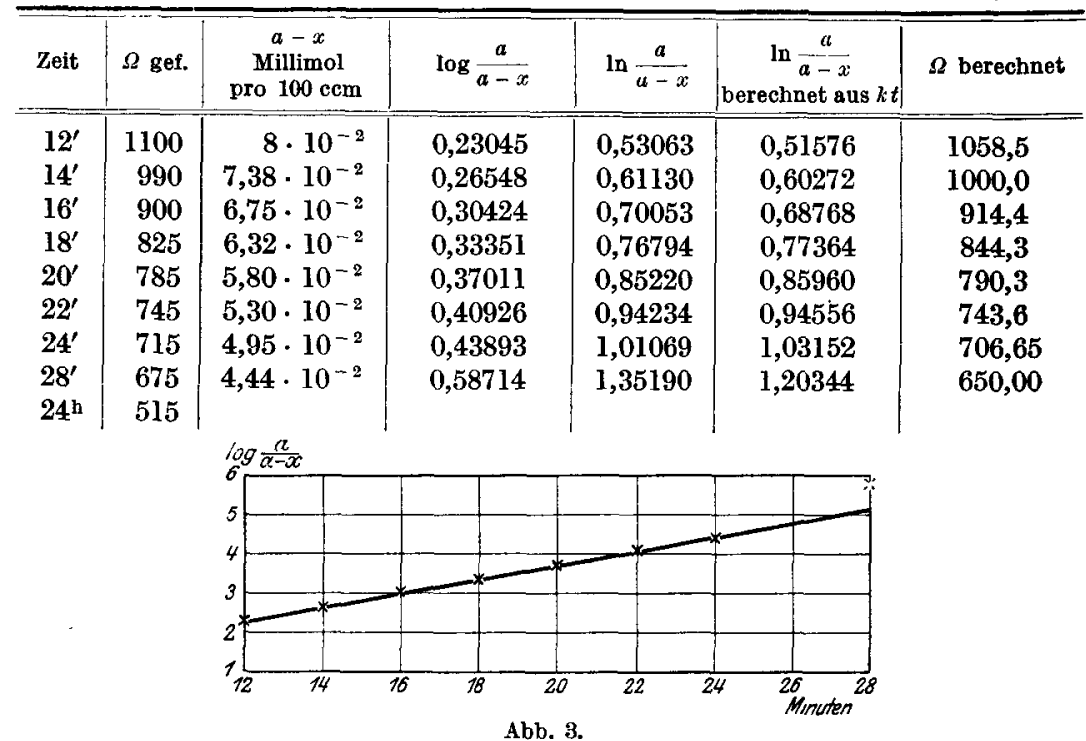

Das Gesetz stimmt im mittleren Teil der Kurve sehr gut mit der experimentell ermittelten überein. Im Anfang der Kurve finden sich größere Abweichungen, weil hier die Widerstandsmessung nicht so genau sein kann, einerseits der schnellen Änderung, andererseits der geringen Konzentration wegen, wodurch unscharfe Minima entstehen; kleine Ungenauigkeit in der Zeitmessung muß hier schon große Fehler zeitigen. Es wurden deshalb für die Berechnung der Konstante der Formel nur die Werte für 12 bis 28 Minuten (bis etwa $70 \%$ Umsatz) benutzt.

Es waren in der Lösung $(100 \mathrm{ccm}) 13,6 \cdot 10^{-2}$ Millimol. Gift. Wären alle Ds-Moleküle gespalten, so wären $2 \cdot 13,6 \cdot 10^{-2}$ Millimol. Cl vorhanden, entsprechend einem Widerstande von $454 \Omega$. Tatsächlich findet sich nach 24 Stunden $475 \Omega$. Es ist also alles Gift gespalten.

Daß das Ds durch Wasser vollkommen gespalten wird, zeigen auch folgende Versuche:

5. Versuch. 0,0216 $\mathrm{g}$ Ds in $1 \mathrm{ccm}$ Alkohol, dann in $70 \mathrm{ccm}$ Leitfähigkeitswasser gelöst. Temp. $19^{\circ}$ C. Widerstandskapazität des Gefäßes 0,47. $-70 \mathrm{ccm}$ 
enthalten $0,0216 \mathrm{~g}$, d. h. $13,6 \cdot 10^{-2}$ Millimol. Ds. Wäre alles gespalten, so wären $\frac{2 \cdot 136 \cdot 10^{-5}}{70} \mathrm{MolCl} / \mathrm{ccm}=\eta$ in der Lösung, entsprechend einem Widerstand von $319 \Omega$. Tatsächlich finden sich nach 24 Stunden $361 \Omega$, nach 48 Stunden $330 \Omega$. Es ist also (fast) alles gespalten.

6. Ver s uch. $0,022 \mathrm{~g}$ Ds in $1 \mathrm{ccm}$ Alkohol gelöst, verdünnt mit 200, 100, 50, 40, 30, $20 \mathrm{ccm}$ Wasser. Temp. $21^{\circ} \mathrm{C} ; c=0,45$.

\begin{tabular}{c|c|c|c|c|c}
\hline $\begin{array}{c}\text { Dichlor- } \\
\text { athylsulfid g }\end{array}$ & $\begin{array}{c}\text { Leittähigkeits- } \\
\text { wasser ccm }\end{array}$ & $\Omega$ nach 24 b & $\Omega$ nach $48 \mathrm{~h}$ & $\begin{array}{c}\Omega \text { theoretisch } \\
\text { zu erwarten }\end{array}$ & $\%$ zerlegt \\
\hline \hline 0,022 & 200 & 875 & 850 & 885 & 100 \\
0,022 & 100 & 440 & 427 & 440 & 100 \\
0,022 & 50 & 233 & 227 & 220 & 100 \\
0,022 & 40 & 186 & 180 & 184 & 100 \\
0,022 & 30 & 160 & 140 & 143 & 100 \\
0,022 & 20 & 114 & 103 & 90 & 100 (fast) \\
0,022 & 10 & 58,5 & 57,0 & 46,8 & 100 (fast)
\end{tabular}

Tetrachlordiäthylsulfid.

1 Tropfen $(0,0238 \mathrm{~g})$ Tchlds wurde in $1 \mathrm{ccm} 90$ proz. Alkohol gelöst, zu $19 \mathrm{ccm}$ Leitfähigkeitswasser im Elektrodengefäß (Kapacität 0,57) zugefügt. Der Widerstand des Wassers von ca. $50000 \Omega$ sinkt nach Einbringen des Giftes schnell und ist nach 2 Minuten $=1140 \Omega$ (vgl. Abb. 4). Den Verlauf der Spaltung zeigt nebenstehende Tabelle:

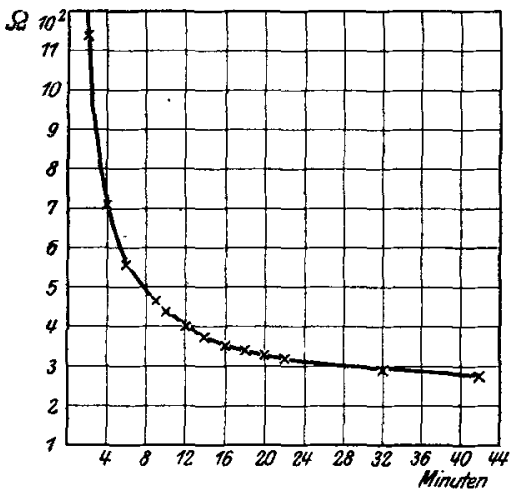

Abb. 4.

\begin{tabular}{r||r|r|r}
\hline Zeit & $\begin{array}{c}\text { Wider- } \\
\text { stand } \Omega\end{array}$ & $\begin{array}{c}\text { Leitfähig- } \\
\text { keit } \\
* 10^{\prime}\end{array}$ & $\begin{array}{c}\text { Mol HCl } \\
\text { pro/ccm } \\
10^{\circ} \eta\end{array}$ \\
\hline $2^{\text {h } 25^{\prime}}$ & 1140 & 5,0 & 1,31 \\
$27^{\prime}$ & 710 & 8,0 & 2,1 \\
$29^{\prime}$ & 555 & 10,3 & 2,7 \\
$32^{\prime}$ & 465 & 12,3 & 3,2 \\
$33^{\prime}$ & 435 & 13,1 & 3,4 \\
$35^{\prime}$ & 400 & 14,3 & 3,7 \\
$37^{\prime}$ & 375 & 15,2 & 4,0 \\
$39^{\prime}$ & 355 & 16,1 & 4,2 \\
$41^{\prime}$ & 340 & 16,8 & 4,4 \\
$43^{\prime}$ & 328 & 17,4 & 4,6 \\
$45^{\prime}$ & 318 & 17,9 & 4,7 \\
$55^{\prime}$ & 293 & 19,4 & 5,1 \\
$3^{h} 5^{\prime}$ & 280 & 20,4 & 5,4 \\
$15^{\prime}$ & 270 & 21,1 & 5,6 \\
$25^{\prime}$ & 265 & 21,5 & 5,7 \\
$20^{h}$ & 190 & 30,0 & 7,9 \\
nach nach weiteren & & & \\
$20^{\text {h }}$ & 190 & 30,0 & 7,9
\end{tabular}

Die Lösung ist $52,7 \cdot 10^{-4}$ molar in bezug auf Gift ; $1 \mathrm{ccm}$ enthalten $52,7 \cdot 10^{-7}$ Mol. Gift.

7. Ver s u ch. $0,018 \mathrm{~g}$ Tetrachlordiäthylsulfid werden in $1 \mathrm{ccm} 90$ proz. Alkobol gelöst, auf $200 \mathrm{ccm}$ mit Leitfähigkeitswasser verdünnt. Gefäßkonstante 0,47. Temp. 
$19^{\circ} \mathrm{C}$. Die Lösung enthält $7,89 \cdot 10^{-7} \mathrm{Mol}$. Gift. Wäre alles zersetzt, so enthielte sie $\frac{4 \cdot 7,89 \cdot 10^{-7}}{200} \mathrm{Mol} \mathrm{Cl} / \mathrm{ccm}=\eta$, entsprechend einem Widerstande von $783 \Omega$. Der Widerstand fällt aber (in 96 Stunden) bis $940 \Omega$. Es sind also $83 \%$ zersetzt.

8. Vers u c h. $0,0187 \mathrm{~g}$ Tetrachlordiäthylsulfid werden in $1 \mathrm{ccm} 90$ proz. Alkohol gelöst, auf $100 \mathrm{ccm}$ mit Leitfähigkeitswasser verdünnt. Temp. $19^{\circ} \mathrm{C}$. Gefäßkonstante 0,47. - Die Lösung enthält 8,2 $10^{-7} \mathrm{Mol} / \mathrm{ccm}$ Gift, entsprechend $32 \cdot 8 \cdot 10^{-7} \mathrm{Cl}$ Mol/ccm $(\eta)$, entsprechend $380 \Omega$. Tatsächlich findet sich nach 48 Stunden ein Widerstand von $500 \Omega$. Es sind also $76 \%$ zersetzt.

9. Versuch. 0,0094 $\mathrm{g}$ Tetrachlordiäthylsulfid werden in $1 \mathrm{ccm} 90$ proz. Alkohol, dann in $50 \mathrm{ccm}$ Leitfähigkeitswasser gelöst. Es befänden sich in der Lösung $32 \cdot 8 \cdot 10^{-7} \mathrm{Mol} / \mathrm{ccm} \mathrm{Cl}$ (entsprechend $380 \Omega$ ), wenn alles Gift zersetzt wäre. Tatsächlich finden sich nach 48 Stunden $548 \Omega$, nach 72 Stunden $485 \Omega$. Es sind also zirka $75 \%$ zersetzt.

\section{Thiodiglykolacetat.}

Ca. $2 \mathrm{cg}$ werden in $1 \mathrm{ccm}$ Alkohol gelöst, zu $19 \mathrm{ccm}$ Leitfähigkeitswasser zugefügt. Temp. $19^{\circ} \mathrm{C}$. Der Widerstand ändert sich nicht meßbar. Er beträgt zuerst ca. $50000 \Omega$, nach ca. $24^{\text {h }} 17000 \Omega$.

\section{Thiodiglykol.}

Ca. $2 \mathrm{cg}$ werden in $1 \mathrm{ccm}$ Alkohol gelöst, zu $19 \mathrm{ccm}$ Leitfähigkeitswasser zugefügt. Temp. $19^{\circ} \mathrm{C}$. Der Widerstand ändert sich nicht und beträgt noch nach ca. $48^{\mathrm{h}}$ ca. $50000 \Omega$.

\section{Dioxäthylsulfon.}

1 Tropfen in $1 \mathrm{ccm} 90$ proz. Alkohol zu $19 \mathrm{ccm}$ Leitfähigkeitswasser. - Temp. $19^{\circ} \mathrm{C}$. Der Widerstand ändert sich nicht und beträgt noch nach $72^{\text {h }}$ ca. $50000 \Omega$.

\section{Diphenylarsinchlorid.}

Diphenylarsinchlorid (DA) wird von Wasser äußerst schnell (momentan) zerlegt.

Versuch: $0,1 \mathrm{~g}$ DA werden in $10 \mathrm{ccm}$ abs. Alkohol gelöst, aus dieser Lösung $1,0 \mathrm{ccm} 20 \mathrm{ccm}$ Leitfähigkeitswasser zugefugt (sofortige Ausscheidung von Diphenylarsinoxyd).

$\begin{array}{rr}\text { Widerstand vor dem Zufügen von DA } & 40000 \Omega \\ \text { nach } 30^{\prime \prime} & 500 ", \\ 1^{\prime} & 400 ", \\ 2^{\prime} & 400 ", \\ 3^{\prime} & 400 ", \\ 4^{\prime} & 400 ", \\ 5^{\prime} & 400 ", \\ 6^{\prime} & 400 ", \\ 7^{\prime} & 400 ", \\ 8^{\prime} & 400 ", \\ 9^{\prime} & 400 ", \\ 10^{\prime} & 400 ",\end{array}$

Der Widerstand bleibt auch nach Stunden unverändert. 


\section{Perchlorierter Ameisensäuremethylester (PA).}

PA wird von Wasser äußerst schnell (momentan) zerlegt.

Ve $\mathbf{r}$ s u ch: $0,3 \mathrm{ccm}$ PA werden in $10 \mathrm{ccm}$ Aceton gelöst, $0,5 \mathrm{ccm}$ der Lösung $20 \mathrm{com}$ Leitfähigkeitswasser zugefügt.

Widerstand vor dem Zufügen von PA $40000 \Omega$

\begin{tabular}{|c|c|c|}
\hline nach $1^{\prime}$ & & \\
\hline $\mathbf{2}^{\prime}$ & & 2 \\
\hline $3^{\prime}$ & & 2 \\
\hline $4^{\prime}$ & & 2 \\
\hline $5^{\prime}$ & & 2 \\
\hline $6^{\prime}$ & & 2 \\
\hline $7^{\prime}$ & & 2 \\
\hline $8^{\prime}$ & & 2 \\
\hline $\mathbf{9}^{\prime}$ & & 2 \\
\hline $10^{\prime}$ & & 2 \\
\hline
\end{tabular}

Der Widerstand bleibt unverändert.

Werden 5 Tropfen PA in $10 \mathrm{ccm}$ Aceton gelöst und von dieser Lösung 2 Tropfen $10 \mathrm{ccm}$ dest. Wasser zugefügt, das vorher mit Tropaeolin 00 versetzt war, so entsteht sofort eine intensive Rotfärbung.

\section{Jodessigester.}

Jodessigester spaltet unter Einwirkung des Wassers (bei Zimmertemperatur) selbst nach längerer Zeit keine Säure ab.

Versu ch: $0,1 \mathrm{ccm}$ Jodessigester werden 2 Stunden lang mit $20 \mathrm{ccm}$ Leitfähigkeitswasser geschüttelt, die wasserklare Flüssigkeit von den öligen Partikelchen abfiltriert. Das Filtrat ist vollkommen neutral (blaues Lackmuspapier bleibt unverändert, Methylorange gelb, Neutralrot violett), hat gar keine Reizwirkung. Gibt intensive Stärkereaktion wie auch Fällung mit Silbernitrat.

Im Leitfähigkeitsapparat ist eine wenn auch nicht starke, doch deutliche Zunahme der Leitfähigkeit zu beobachten.

Versuch: Wurde 0,1 ccm Jodessigester zu $20 \mathrm{ccm}$ Leitfähigkeitswasser zugesetzt, so sank der Widerstand von $40000 \Omega$ nach 2 Stunden auf 8000 , nach weiteren 24 Stunden auf $3600 \Omega$.

Zur Erklärung dieser Beobachtungen könnte man annehmen, daß die unter der Einwirkung des Wassers möglicherweise zunächst entstehende Jodwasserstoffsäure weiter unter Entstehung von Essigester und Glykolsäureester nach der Gleichung wirkt:

$$
=c \begin{gathered}
2 \mathrm{CH}_{2} \mathrm{~J} \mathrm{COOC}_{2} \mathrm{H}_{5}+2 \mathrm{H}_{2} \mathrm{O} \\
=\mathrm{CH}_{3} \cdot \operatorname{COOCC}_{2} \mathrm{H}_{5}+\mathrm{CH}_{2} \mathrm{OHCOOC}_{2} \mathrm{H}_{5}+\mathrm{J}_{2}+\mathrm{H}_{2} \mathrm{O} .
\end{gathered}
$$

Folgende Annahme ist jedoch wohl richtiger. Eine geringe Menge (infolge Lichteinwirkung usw.) abgespaltenes Jod wird in Jodessigester gelöst und wird dann ganz allmählich an das Wasser abgegeben. Dies würde die allmähliche Zunahme der Leitfähigkeit wie auch das Vorhandensein von Jod in der Lösung einfach erklären. 
Zur Entscheidung dieser Frage wurde in dem Wasser zur Erhöhung der Jodaufnahmefähigkeit Jodkalium gelöst und diese Lösung mit dem Jodessigester einige Male durchgeschüttelt. Es trat sofort Jodreaktion auf. Der Vorgang wurde dann quantitativ verfolgt, und zwar wurden $25 \mathrm{ccm}$ feldmäßiges jodessigsaures Äthyl mit̂ $75 \mathrm{ccm} 5$ proz. Jodkaliumlösung verschieden lange geschüttelt und nachher das in die Jodkaliumlösung übergegangene Jod mit $1 / 100$ n-Thiosulfat titriert.

$$
\begin{aligned}
& \text { Dauer des Schüttelns . . . . } 10^{\prime} 2 \text { Std. } 4 \text { Std. } 6 \text { Std. } 24 \text { Std. } 48 \text { Std. } \\
& \text { pro } 25 \mathrm{ccm} \mathrm{KJ} \text {-Lösung ver- } \\
& \text { brauchte } \mathrm{ccm} 1 / 100 \mathrm{n} \text {-Thio- }
\end{aligned}
$$

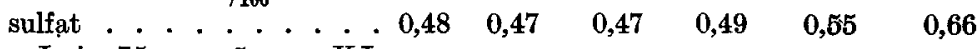

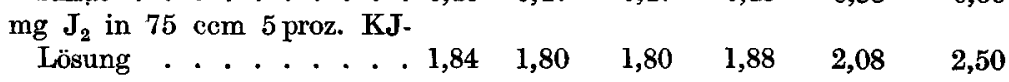

Bei Anwendung von $75 \mathrm{ccm} 10 \mathrm{proz}$. KJ-Lösung wurden 2,50 mg $J_{2}$ in $10^{\prime}$ erhalten.

Wie aus der Tabelle ersichtlich, wurde nach sehr langem Schütteln in der Jodkaliumlösung etwas mehr Jod gefunden als am Anfang, so daß man auf eine geringe Zersetzung schließen könnte. Es sei aber bemerkt, daß auf die Temperatur, die das Gleichgewicht stark beeinflußt, nicht geachtet worden ist. Gegen eine Zersetzung mit Wasser spricht die Tatsache, daß Jodessigester gegen Alkali außerordentlich beständig ist. Außerdem wurde zufällig vorhandenes reines bromessigsaures Äthyl mit Jodkaliumlösung geschüttelt. Es war keine Abgabe von Brom bemerkbar.

Aus diesen Versuchen ist zu schließen, daß eine kleine Menge elementares Jod in dem technischen Jodessigester vorgebildet ist, das bei Berührung mit Wasser in dieses übergeht. Möglich ist auch eine allmählich fortschreitende Abspaltung von Jod in dem Jodessigester. Soviel geht jedenfalls aus den Untersuchungen mit Bestimmtheit hervor, daß die Berührung mit Wasser eine Zersetzung des Jodessigesters nicht herbeiführt und auch nicht beschleunigt.

\section{Jodaceton.}

Jodaceton wird von Wasser (praktisch) nicht zerlegt. Die geringfügige Zunahme der Leitfähigkeit rührt wohl von Verunreinigungen des etwas zersetzten Präparates her.

Vers uch: Wurden $0,1 \mathrm{ccm}$ Jodaceton $20 \mathrm{ccm}$ Leitfähigkeitswasser zugefügt, so sinkt der Widerstand von $40000 \Omega$ nach einer Stunde erst auf $30000 \Omega$ und beträgt selbst nach 24 Stunden $7000 \Omega$. Die wasserklare Flüssigkeit hat eine neutrale Reaktion, gibt mit Stärke keine Blaufärbung (enthält keine Jodmolekeln), nach Zusatz von ${ }^{\mathrm{AgNO}_{3}}$ entsteht jedoch eine Fällung; die Flüssigkeit enthält demnach Jodionen. 


\section{Benzyljodid.}

Benzyljodid wird von Wasser, wenn überhaupt, so doch nur äußerst langsam zerlegt. Möglicherweise beruht die beobachtete langsame Zunahme der Leitfähigkeit auf Verunreinigungen des etwas zersetzten Präparates.

Ve r s uch: $1,0 \mathrm{ccm}$ Benzyljodid wird in $10 \mathrm{ccm}$ abs. Alkohol gelöst, $0,5 \mathrm{ccm}$ dieser Lösung werden $20 \mathrm{ccm}$ Leitfähigkeitswasser zugesetzt.

Widerstand vor dem Zufügen von Benzyljodid $35000 \Omega$

$\begin{array}{cr}\text { nach } 1^{\prime} & 25000, " \\ 10^{\prime} & 20000, " \\ 25^{\prime} & 20000, " \\ 40^{\prime} & 16000, " \\ 90^{\prime} & 12000, " \\ 140^{\prime} & 10000, \\ 24 \text { Std. } & 3100,\end{array}$

Die wasserklare Flüssigkeit ist neutral, gibt keine Stärkereaktion, aber mit $\mathrm{AgNO}_{3}$ eine schwache Trübung, sie enthält also geringe Mengen Jodionen.

\section{Benzylbromid.}

Benzylbromid wird von Wasser schneller als Benzyljodid zersetzt.

V e r s uch: $1,0 \mathrm{ccm}$ Benzylbromid werden in $10 \mathrm{ccm}$ abs. Alkohol gelöst, davon $0,5 \mathrm{ccm} 20 \mathrm{ccm}$ Leitfähigkeitswasser zugefügt.

Widerstand vor dem Zufügen von Benzylbromid $35000 \Omega$

$\begin{array}{cr}\text { nach } \mathbf{1}^{\prime} & 22000, \\ 2^{\prime} & 20000 ", \\ 10^{\prime} & 10000 ", \\ 25^{\prime} & 5000 ", \\ 45^{\prime} & 2700 ", \\ 95^{\prime} & 2000 ", \\ 24 \text { Stdn. } & 280, "\end{array}$

Die wasserklare Flüssigkeit zeigt eine schwach saure Reaktion (Lakmus rot, Methylorange rot) und gibt mit $\mathrm{AgNO}_{3}$ eine Fällung, enthält demnach Bromionen. Wie Benzylbromid verhält sich X ylylbromid.

Von den folgenden 4 Stoffen wurde jedesmal $0,5 \mathrm{ccm}$ auf $10 \mathrm{ccm}$ absoluten Alkohol gelöst, von dieser Lösung 0,5 ccm zu $20 \mathrm{ccm}$ Leitfähigkeitswasser zugesetzt. Die Resultate waren folgende:

\section{Dibrommethyläther.}

Widerstand vor Zufügen der Giftlösung ca. $50000 \Omega ; 20^{\prime \prime}$ danach $42 \Omega$; auf dieser Höhe blieb der Widerstand unverändert stehen. Die Flüssigkeit war stark sauer geworden. Zersetzung also sehr schnell (momentan).

\section{Dichlormethyläther.}

Widerstand vorher ca. $40000 \Omega ; 30^{\prime \prime}$ nach Zufügen der Dichlormethylätherlösung $60 \Omega$; bleibt unverändert.

Flüssigkeit stark sauer. - Also gleichfalls momentane Zersetzung. 


\section{Dibrommethylsulfid.}

Widerstand ca. $30000 \Omega$.

\begin{tabular}{|c|c|c|c|c|c|}
\hline Nach & $20^{\prime \prime}$ & 250 & $\Omega$ nach & $\mathbf{3}^{\prime}$ & $110 \Omega$ \\
\hline & $30^{\prime \prime}$ & 200 & , & $4^{\prime}$ & 90 \\
\hline & $40^{\prime \prime}$ & 170 & , & $5^{\prime}$ & 87 \\
\hline & $50^{\prime \prime}$ & 160 & ", & $11^{\prime}$ & 65 \\
\hline & $60^{\prime \prime}$ & 150 & ,, & $25^{\prime}$ & 47 \\
\hline & $120^{\prime \prime}$ & 120 & ," & $45^{\prime}$ & 43 \\
\hline
\end{tabular}

Widerstand bleibt unverändert bei $\mathbf{4 3} \Omega$; Flüssigkeit stark sauer Zersetzung also schnell, aber von meßbarer Geschwindigkeit.

\section{Dichlormethylsulfid.}

Widerstand vorher ca. $30000 \Omega$.

\begin{tabular}{|c|c|c|c|c|c|}
\hline Nach & $20^{\prime \prime}$ & $1000 \Omega$ & nach & $4^{\prime}$ & 105 \\
\hline & $45^{\prime \prime}$ & 400 & & $5^{\prime}$ & 95 \\
\hline & $60^{\prime \prime}$ & 300, & & $6^{\prime}$ & 90 \\
\hline & $90^{\prime \prime}$ & 200 & & $7^{\prime}$ & 85 \\
\hline & $120^{\prime \prime}$ & 160 & & $9^{\prime}$ & 80 \\
\hline & $150^{\prime \prime}$ & 130, & & $11^{\prime}$ & 76 \\
\hline & $180^{\prime \prime}$ & 120, & & $15^{\prime}$ & 74 \\
\hline & $210^{\prime \prime}$ & 110, & & $17^{\prime}$ & 72 \\
\hline
\end{tabular}

Widerstand bleibt weiterhin unverändert. Flüssigkeit stark sauer. Zersetzung also schnell, aber von gut meßbarer Geschwindigkeit.

\section{Dibromäthylsulfid.}

$0,1 \mathrm{~g}$ wurden in $10 \mathrm{ccm}$ absolutem Alkohol gelöst, von dieser Lösung $1 \mathrm{ccm}$ zu $20 \mathrm{ccm}$ Leitfähigkeitswasser gefügt. Widerstand vorher ca. $40000 \Omega$.

$\begin{array}{rrr}\text { Nach } 30^{\prime \prime} & 1000 \Omega & \text { nach } 4^{\prime} 300 \Omega \\ 60^{\prime \prime} & 400 ", & 5^{\prime} 290 \% \\ 120^{\prime \prime} & 300 ", & 6^{\prime} 290 ”, \\ 180^{\prime \prime} & 300, " & \text { bleibt unverändert. }\end{array}$

Flüssigkeit stark sauer. - Zersetzungsgeschwindigkeit groß, aber noch meßbar.

Äthylen-bis- $\left(\omega\right.$-Chloräthyl-) sulfid $\left(\mathrm{ClC}_{2} \mathrm{H}_{4} \cdot \mathrm{S} \cdot \mathrm{C}_{2} \mathrm{H}_{4} \cdot \mathrm{S} \cdot \mathrm{C}_{2} \mathrm{H}_{4} \mathrm{Cl}\right)$.

$0,05 \mathrm{~g}$ in $10 \mathrm{ccm}$ absolutem Alkohol gelöst, von der Lösung $1 \mathrm{ccm}$ zu $20 \mathrm{ccm}$ Leitfähigkeitswasser gefügt. Widerstand vorher ca. $30000 \Omega$.

\begin{tabular}{|c|c|c|c|c|c|}
\hline Nach & $1 / 2^{\prime}$ & 17000 & nach & $8^{\prime}$ & 4500 \\
\hline & $1^{\prime \prime}$ & 16000 & & $12^{\prime}$ & 2800 \\
\hline & $2^{\prime}$ & 15000 & & $18^{\prime}$ & 2000 \\
\hline & $3^{\prime}$ & 13000 & & $21^{\prime}$ & 1800 \\
\hline & $4^{\prime}$ & 9000 & & $25^{\prime}$ & 1650 \\
\hline & $5^{\prime}$ & 8000 & & $40^{\prime}$ & 1150 \\
\hline & $6^{\prime}$ & 6000 & & $50^{\prime}$ & 1000 \\
\hline & $7^{\prime}$ & 5000 & & $60^{\prime}$ & 930 \\
\hline
\end{tabular}


Flüssigkeit stark sauer. Die Zersetzung durch Wasser erfolgt also sehr langsam.

Bromäthyläthylsulfid $\left(\mathrm{BrCH}_{2}, \mathrm{CH}_{2} \cdot \mathrm{S} \cdot \mathrm{C}_{2} \mathrm{H}_{5}\right)$.

$0,5 \mathrm{ccm}$ der Substanz wurden in $10 \mathrm{ccm}$ abs. Alkohol gelöst, von dieser Lösung $0,5 \mathrm{ccm} 20 \mathrm{ccm}$ Leitfähigkeitswasser zugefügt.

Widerstand vorher $30000 \Omega$.

$\begin{array}{ccccc}\text { Nach } & 20^{\prime \prime} & 400 \Omega & \text { nach } 1^{1} / 2^{\prime} & 200 \Omega \\ 30^{\prime \prime} & 350, & 2^{\prime} & 190 " \\ 50^{\prime \prime} & 300 ", & 4^{\prime} & 170 " \\ 60^{\prime \prime} & 250 \# & 5^{\prime} & 160 "\end{array}$

Der Widerstand bleibt unverändert. Bromäthyläthylsulfid wird demnach durch Wasser schnell zersetzt.

\section{Zusammenfassung.}

Folgende Kampfstoffe werden durch Wasser zersetzt:

Phosgen äußerst rasch (momentan),

Perchlorierter Ameisensäuremethylester äußerst rasch (momentan).

Diphenylarsinchlorid äußerst rasch (momentan),

Dichlormethyläther äußerst rasch (momentan),

Dibrommethyläther äußerst rasch (momentan),

Dibrommethylsulfid schnell, aber gut meßbar,

Dichlormethylsulfid schnell, aber gut meßbar,

Dibromäthylsulfid schnell, aber gut meßbar,

Dichloräthylsulfid allmählich,

Tetrachlordiäthylsulfid allmählich,

Äthylen-bis-( $\omega$-Chloräthyl-)sulfid langsam,

Benzylbromid sehr langsam,

Xylylbromid sehr langsam,

Benzyljodid sehr langsam.

Folgende Kampfstoffe werden durch Wasser (praktisch) nicht zersetzt:

Chlorpikrin,

Jodessigester,

Jodaceton.

Bei der ersten Gruppe kann eine Säurebildung stets nachgewiesen werden. (Eine Ausnahme bildet vielleicht Benzyljodid, dessen Zersetzung durch Wasser möglicherweise durch die nicht genügende Reinheit des Präparates vorgetäuscht wird.) Die Zunahme der Länge der Kohfenstoffkette, wie auch der Ersatz von Sauerstoff durch Schwefel im Molekül erhöhen die Beständigkeit gegen Wasser. 